\title{
On the Families of Gibbs Semigroups
}

\author{
V. A. Zagrebnov \\ Laboratory of Theoretical Physics, Joint Institute for Nuclear Research, SU-141980 Dubna, USSR
}

\begin{abstract}
The families of Gibbs semigroups with generators from conveniently bounded monotonous families of self-adjoint operators are proved to be compact in the trace-norm topology.
\end{abstract}

\section{Introduction}

Let $\mathscr{H}$ be a separable Hilbert space and $\mathscr{C}_{p}(\mathscr{H})$ be the Banach space of compact operators on $\mathscr{H}$ with finite $p$-norm:

$$
\|A\|_{p}=\left(\sum_{n=1}^{\infty}\left(\lambda_{n}(A)\right)^{p}\right)^{1 / p}, \quad 1 \leqq p<\infty .
$$

Here $\left\{\lambda_{n}(A)\right\}_{n=1}^{\infty}$ are the singular values of the operator $A \in \mathscr{C}_{p}(\mathscr{H})$. Then one has: $\mathscr{C}_{1}(\mathscr{H})$ (trace-class) $\subset \mathscr{C}_{2}(\mathscr{H})$ (Hilbert-Schmidt operators) $\subset \ldots \subset \mathscr{C}_{p}(\mathscr{H}) \subset \ldots \subset \mathscr{C}_{\infty}(\mathscr{H})$ (compact operators) $\subset \mathscr{L}(\mathscr{H})$ (bounded operators) [1].

Strongly continuous one-parameter self-adjoint semigroups $\{Q(t)\}$, $Q: \mathbb{R}_{+}^{1} \cup\{0\} \rightarrow \mathscr{L}(\mathscr{H})$, with the values in $\mathscr{C}_{1}(\mathscr{H})$ for $t \in \mathbb{R}_{+}^{1}$, arise naturally in quantum statistical mechanics. Usually they are called Gibbs semigroups $[2,3]$. However, in some cases they are not to be self-adjoint (e.g. for $t \in \mathbb{C}_{+}$, the complex right half-plane $\{z \in \mathbb{C}: \operatorname{Re} z>0\}$, or see Example 1). Hence, more relevant is the following

Definition 1. A strongly continuous semigroup $Q(t)$ in a separable Hilbert space is called a Gibbs semigroup if $Q(t): \mathbb{R}_{+}^{1} \rightarrow \mathscr{C}_{1}(\mathscr{H})$.

Every strongly continuous semigroup $Q(t)$ is known (see e.g. [4, X.8] or $[5, I X, \S 1])$ to be generated by a closed densely defined quasi- $m$-accretive operator $T$ (semigroup generator) and has the form: $Q(t)=\exp (-t T)$.

Example 1. Quasi-m-accretive generators arise in perturbation theory of Gibbs semigroups $[3,6-8]$. Let $T$ be a positive generator of Gibbs semigroup. Suppose that $U$ is a symmetric $T$-bounded operator with $T$-bound $b<1$, i.e. 
$\|U \psi\| \leqq a\|\psi\|+b\|T \psi\|$ for arbitrary vector $\psi$ in the domain $D(T)$ of the operator $T$. Then the operator $H_{\lambda}=T+\lambda U$ is well defined on $D(T)$ for $\left\{\lambda \in \mathbb{C}:|\lambda|<b^{-1}\right\}$ as algebraic sum. This operator is an $m$-sectorial generator of Gibbs semigroup because for $\psi \in D(T)$ the values of the quadratic form $h_{\lambda}[\psi]=\left(\psi, H_{\lambda} \psi\right)$ lie in the sector

$$
S_{\gamma, \omega}=\{z \in \mathbb{C}:|\arg (z-\gamma)|<\omega\}
$$

where $\gamma=-a|\lambda|\left(1-b^{2}|\lambda|^{2}\right)^{-3 / 2}$ and $\omega=\operatorname{arctg}\left(\sqrt{1-b^{2}|\lambda|^{2}} / b|\lambda|\right)$.

For some systems of quantum statistical mechanics the Gibbs semigroups are defined as limits of semigroup sequences corresponding to suitable families of Hamiltonians (generators). The aim of this paper is to describe a connection between compactness of Gibbs semigroup families in $\|\cdot\|_{1}$-topology and the properties of the corresponding generator familes. Therefore, our strategy here is to turn a relatively weak (or easily checked) information about the generator families into a stronger information about the semigroups. The present paper is restricted to the well-known particular case of the Gibbs semigroups with selfadjoint generators. A possible generalization to the quasi- $m$-accretive generators will be published elsewhere.

In Sect. 2 we set up some general conditions for a sequence of the self-adjoint generators to provide the $\|\cdot\|_{1}$-convergence of the corresponding Gibbs semigroups. In Sect. 3 we shall define for the families of self-adjoint operators a notion (monotonous family), which will prove to be useful in our discussion of the Gibbs semigroups $\|\cdot\|_{1}$-compactness for $t \in \mathbb{C}_{+}$. In particular, by analytic continuation arguments the $\|\cdot\|_{1}$-compactness in $\mathbb{C}_{+}$of the Gibbs semigroup families as families of operator-valued functions $\|\cdot\|_{1}$-holomorphic on the right half-plane $\mathbb{C}_{+}$is established. We conclude Sect. 3 with two examples from statistical mechanics of systems with highly singular two-body potentials.

\section{Preliminary Statements}

This section starts with a statement which further allows us to "lift" the convergence of the Gibbs semigroup sequences in the strong operator topology to convergence in $\|\cdot\|_{1}$-norm.

Proposition 1. Let $B \in \mathscr{C}_{\infty}(\mathscr{H})$ and $B \geqq 0$. Let $\left\{A_{k}\right\}_{k=1}^{\infty}$ be a sequence of bounded and self-adjoint operators. Suppose that

$$
-B \leqq A_{k} \leqq B
$$

and $A_{k} \stackrel{s}{\longrightarrow} A$ (strongly). Then operator $A \in \mathscr{C}_{\infty}(\mathscr{H})$ is self-adjoint and $A_{k} \stackrel{\|\cdot\|}{\longrightarrow} A$ (in operator norm).

Proof. The first part of the proposition follows from the strong convergence $A_{k} \stackrel{s}{\longrightarrow} A$, inequalities $\left\{-B \leqq A_{k} \leqq B\right\}_{k=1}^{\infty}$ and the Weyl's min-max principle. Therefore, suffice it to consider the case $A=0$. Suppose that (for a subsequence) one has $\left\|A_{m}\right\| \geqq a>0$. Then there are (note that $A_{m}, m=1,2, \ldots$, are compact by the above arguments) such sequences $\left\{b_{m}\right\}_{m=1}^{\infty}$ and $\left\{u_{m}\right\}_{m=1}^{\infty} \in \mathscr{H}$ that $\left\|u_{m}\right\|=1$, $m=1,2, \ldots$ and $A_{m} u_{m}=b_{m} u_{m},\left|b_{m}\right| \geqq a$. 
Then it follows that $\left\|B^{1 / 2} u_{m}\right\| \geqq a^{1 / 2}$. Note, that the operator $B^{1 / 2}$ is also compact, therefore, one can take in $\mathscr{H}$ a subspace $M$ such that $\operatorname{dim} M<\infty$ and

$$
\left\|B^{1 / 2} u\right\| \leqq 2^{-1} a^{1 / 2}\|u\|
$$

for $u \in M^{\perp}$. Let $P_{M}$ be the projection on $M$. Then from the above inequality and the estimate

$$
\left\|B^{1 / 2} u_{m}\right\| \leqq\left\|B^{1 / 2} P_{M} u_{m}\right\|+\left\|B^{1 / 2}\left(1-P_{M}\right) u_{m}\right\|
$$

one sees that $\left\|B^{1 / 2}\right\|\left\|P_{M} u_{m}\right\| \geqq\left\|B^{1 / 2} P_{M} u_{m}\right\| \geqq 2^{-1} a^{1 / 2}$. Hence, $\left\|P_{M} u_{m}\right\|$ $\geqq 2^{-1} a^{1 / 2}\left\|B^{1 / 2}\right\|^{-1}$ and

$$
\left\|P_{M} A_{m} u_{m}\right\| \geqq 2^{-1} a^{3 / 2}\left\|B^{1 / 2}\right\|^{-1} .
$$

On the other hand, $\left\|A_{m} P_{M}\right\| \rightarrow 0$ and hence $\left\|P_{M} A_{m}\right\| \rightarrow 0$ in contradiction with the above inequality.

Now we can prove the following important for our analysis.

Lemma. Let $\left\{T_{k}\right\}_{k=1}^{\infty}$ and $T$ be self-adjoint operators in $\mathscr{H}$.

Assume :

(i) there exists a self-adjoint operator $T_{-}$such that

(i') $T_{-} \leqq T_{k}$,

(i") $\exp \left(-t T_{-}\right) \in \mathscr{C}_{p}(\mathscr{H})$ for $t \in \mathbb{R}_{+}^{1}$ and $1 \leqq p<\infty$;

(ii) the sequence $\left\{T_{k}\right\}_{k=1}^{\infty}$ converges to the operator $T$ in the strong generalized sense. Then one has for $t \in \mathbb{R}_{+}^{1}$ :

(a) $Q(t)=\exp (-t T) \in \mathscr{C}_{1}(\mathscr{H})$

(b) $Q(t)=\|:\|_{1}-\lim _{k \rightarrow \infty} \exp \left(-t T_{k}\right)$.

Proof. (a) From definition of the Banach space $\mathscr{C}_{p}(\mathscr{H})$ and assumption $\left(\mathrm{i}^{\prime \prime}\right)$ it follows that the operator $T_{-}$is semibounded from below and has only a point spectrum. Moreover the semigroup property $Q_{-}(t)=\left(\exp \left(-t / p T_{-}\right)\right)^{p}$ implies that $Q_{-}(t) \in \mathscr{C}_{1}(\mathscr{H})$ for $t \in \mathbb{R}_{+}^{1}$. The assumptions (i') and (ii) show that $T_{-} \leqq T$. Then from the Weyl's min-max principle it follows that the operator $T$ also has only a point spectrum with $\mu_{n}\left(T_{-}\right) \leqq \mu_{n}(T)$. Here $\mu_{n}(\cdot)$ is the $n$-th eigenvalue from the bottom of the spectrum counting multiplicity. Thus, one has: $\left\{\exp \left(-t T_{k}\right)\right\}_{k=1}^{\infty} \in \mathscr{C}_{1}(\mathscr{H})$ and $\exp (-t T) \in \mathscr{C}_{1}(\mathscr{H})$ for $t \in \mathbb{R}_{+}^{1}$.

(b) The convergence of the sequence $\left\{T_{k}\right\}_{k=1}^{\infty}$ in the strong generalized sense means that the resolvent $R_{\zeta}(T)=(\zeta-T)^{-1}$ is the strong limit of the sequence $\left\{R_{\zeta}\left(T_{k}\right)\right\}_{k=1}^{\infty}$ for $\{\zeta \in \mathbb{C}: \operatorname{Im} \zeta \neq 0\}$. Since operator $T_{-}$is semibounded from below this convergence takes place also for real $\zeta$ from the set $\left\{\zeta: \zeta<\min _{n} \mu_{n}\left(T_{-}\right)\right\}$. On the other hand, for such $\zeta$ one has:

$$
R_{\zeta}\left(T_{-}\right) \leqq R_{\zeta}\left(T_{k}\right)<0
$$

Therefore, due to Proposition 1 one gets for $\left\{\zeta: \zeta<\min _{n} \mu_{n}\left(T_{-}\right)\right\}$:

$$
R_{\zeta}(T)=\|\cdot\|-\lim _{k \rightarrow \infty} R_{\zeta}\left(T_{k}\right) .
$$

This $\|\cdot\|$-convergence can be easily extended to the whole set $\varrho\left(T_{-}\right)$, i.e. $T_{k} \rightarrow T$ in the norm generalized sense. Then, as a consequence one has for $t \in \mathbb{R}_{+}^{1}$ :

$$
Q(t)=\|\cdot\|-\lim _{k \rightarrow \infty} Q_{k}(t)
$$


where $Q_{k}(t)=\exp \left(-t T_{k}\right)$. Note, that the $\|\cdot\|$-limit (1) and the fact, that the semigroups $Q(t)$ and $\left\{Q_{k}(t)\right\}_{k=1}^{\infty}$ for $t \in \mathbb{R}_{+}^{1}$ are at least in $\mathscr{C}_{\infty}(\mathscr{H})$ provide the convergence of eigenvalues:

$$
\mu_{n}(Q(t))=\lim _{k \rightarrow \infty} \mu_{n}\left(Q_{k}(t)\right)
$$

uniformly in $n \geqq 1$. On the other hand, for all $n, k \geqq 1$ and $t \in \mathbb{R}_{+}^{1}$ there are inequalities : $\mu_{n}\left(Q_{k}(t)\right) \leqq \mu_{n}\left(Q_{-}(t)\right)$, so one gets :

$$
\operatorname{Tr} Q(t)=\lim _{k \rightarrow \infty} \operatorname{Tr} Q_{k}(t)
$$

Now, let $P$ be a finite rank spectral projector of the operator $Q(t)$ for a fixed $t \in \mathbb{R}_{+}^{1}$, then

$$
\begin{aligned}
\left\|Q(t)-Q_{k}(t)\right\|_{1} \leqq & \|Q(t)-P Q(t) P\|_{1} \\
& +\left\|P\left(Q(t)-Q_{k}(t)\right) P\right\|_{1}+\left\|P Q_{k}(t) P-Q_{k}(t)\right\|_{1} .
\end{aligned}
$$

Due to $Q(t) \in \mathscr{C}_{1}(\mathscr{H})$ and the limits (1), (3), for a given $\varepsilon>0$ one can choose $P$ and $N_{\varepsilon}$ such that

$$
\|Q(t)-P Q(t) P\|_{1}<\varepsilon
$$

and

$$
\begin{aligned}
& \left\|P\left(Q(t)-Q_{k}(t)\right) P\right\|_{1}<\varepsilon, \\
& \left|\operatorname{Tr}\left(Q(t)-Q_{k}(t)\right)\right|<\varepsilon
\end{aligned}
$$

for $k>N_{\varepsilon}$. Since the operator $Q_{k}(t) \in \mathscr{C}_{1}(\mathscr{H})$ has the spectral decomposition:

$$
\left(Q_{k}(t) \cdot\right)=\sum_{n=1}^{\infty} \mu_{n}\left(Q_{k}(t)\right)\left(\cdot, e_{n}\left[Q_{k}(t)\right]\right) e_{n}\left[Q_{k}(t)\right],
$$

where $\left\{e_{n}\left[Q_{k}(t)\right]\right\}_{n=1}^{\infty}$ is the complete orthonormal set of $Q_{k}(t)$ 's eigenvectors, one has:

$$
\begin{aligned}
\left\|Q_{k}(t)-P Q_{k}(t) P\right\|_{1} \leqq & 2 \sum_{n=1}^{\infty} \mu_{n}\left(Q_{k}(t)\right) \| e_{n}\left[Q_{k}(t)\right] \\
& -P e_{n}\left[Q_{k}(t)\right] \| \leqq 2\left\{\sum_{n=1}^{\infty} \mu_{n}\left(Q_{k}(t)\right)\right\}^{1 / 2} \\
& \cdot\left\{\sum_{n=1}^{\infty} \mu_{n}\left(Q_{k}(t)\right)\right. \\
& \left.\cdot\left\|e_{n}\left[Q_{k}(t)\right]-P e_{n}\left[Q_{k}(t)\right]\right\|^{2}\right\}^{1 / 2} \\
\leqq & 2\left(\operatorname{Tr} Q_{-}(t)\right)^{1 / 2}\left\{\sum_{n=1}^{\infty} \mu_{n}\left(Q_{k}(t)\right)\right. \\
& \left.-\sum_{n=1}^{\infty} \mu_{n}\left(Q_{k}(t)\right)\left\|P e_{n}\left[Q_{k}(t)\right]\right\|^{2}\right\}^{1 / 2} \\
\leqq & 2\left(\operatorname{Tr} Q_{-}(t)\right)^{1 / 2}\left\{\operatorname{Tr} Q_{k}(t)-\operatorname{Tr} P Q_{k}(t) P\right\}^{1 / 2} .
\end{aligned}
$$


The estimates (6) and (7) show that

$$
\left\|Q_{k}(t)-P Q_{k}(t) P\right\|_{1} \leqq\left(12 \varepsilon \operatorname{Tr} Q_{-}(t)\right)^{1 / 2}
$$

for $t \in \mathbb{R}_{+}^{1}$ and $k>N_{\varepsilon}$. The collection of inequalities (4)-(6) and (8) proves (b).

Remark 1. The above arguments [see the second part of the Proof (b)] actually prove the following.

Proposition 2. Let $\left\{C_{k}\right\}_{k=1}^{\infty} \in \mathscr{C}_{1}(\mathscr{H})$ be a sequence of self-adjoint operators and $C \in \mathscr{C}_{1}(\mathscr{H})$. Assume that:

(i) $C_{k} \stackrel{w}{\longrightarrow} C($ weakly);

(ii) $\left|C_{k}\right| \stackrel{w}{\longrightarrow}|C|$; and

(iii) $\left\|C_{k}\right\|_{1} \rightarrow\|C\|_{1}$.

Then $C=\|\cdot\|_{1}-\lim _{k \rightarrow \infty} C_{k}$.

\section{Principal Results and Examples}

We use here the statements derived in the preceding section for studying the compactness problem for the Gibbs semigroup families in the $\|\cdot\|_{1}$-topology. We are now in a position to introduce a useful notion appropriate for our aim:

Definition 2. A family $\left\{T_{\alpha}\right\}_{\alpha \in A}$ of self-adjoint operators in $\mathscr{H}$ is said to be monotonous if every infinite subfamily contains at least one ordered subsequence $\left\{T_{\alpha_{k}}\right\}_{k=1}^{\infty}$ in the sense that $T_{\alpha_{1}} \leqq T_{\alpha_{2}} \leqq \ldots$ or $T_{\alpha_{1}} \geqq T_{\alpha_{2}} \geqq \ldots$

With this notion we obtain the following

Theorem 1. Let $\left\{T_{\alpha}\right\}_{\alpha \in A}$ be a monotonous family of self-adjoint operators. Assume that:

(i) there is a self-adjoint operator $T_{-}$such that $T_{-} \leqq T_{\alpha}$ and $\exp \left(-t T_{-}\right) \in \mathscr{C}_{p}(\mathscr{H})$ for $t \in \mathbb{R}_{+}^{1}$ and $1 \leqq p<\infty$;

(ii) there is a self-adjoint operator $T_{+}$such that $T_{\alpha} \leqq T_{+}$. Then the corresponding family of the semigroups $\left\{Q_{\alpha}(t)\right\}_{\alpha \in A}$ belongs to $\mathscr{C}_{1}(\mathscr{H})$ and is $\|\cdot\|_{1}$-compact for $t \in \mathbb{R}_{+}^{1}$.

Proof. The monotonicity of a subsequence $\left\{T_{\alpha_{k}}\right\}_{k=1}^{\infty}$ and its boundedness: $T_{-} \leqq T_{\alpha_{k}} \leqq T_{+}$, imply the existence in $\mathscr{H}$ of a self-adjoint operator $T$ that is the strong generalized limit of this subsequence. The rest of the proof easily follows from the lemma.

Remark 2. It is at this point that the self-adjointness of the family $\left\{T_{\alpha}\right\}_{\alpha \in A}$ is most essential. For a general $m$-sectorial-operator sequence one faces with a difficult problem of the limiting operator existence (see [5, VIII, §3]).

Remark 3. To see that Theorem 1 does not extend immediately to all $t \in \mathbb{C}_{+}$, notice that for $\left\{t \in \mathbb{C}_{+}: \operatorname{Im} t \neq 0\right\}$, Eq. (1) is not a consequence of the generalized norm convergence alone. It follows, however, from the semigroup properties by the analytic continuation arguments. 
Theorem 2. Let $\left\{T_{\alpha}\right\}_{\alpha \in A}$ be a family of operators as in Theorem 1. Then the Gibbs semigroups $\left\{Q_{\alpha}(t)\right\}_{\alpha \in A}$ form on $\mathbb{C}_{+} a\|\cdot\|_{1}$-holomorphic operator-valued family of the functions which is $\|\cdot\|_{1}$-compact in this domain.

Proof. Every $Q_{\alpha}(t)$ can be continued from $\mathbb{R}_{+}^{1}$ to a strongly holomorphic Gibbs semigroup on the domain $\mathbb{C}_{+}$by means of the Dunford-Taylor formula (see e.g. $[5, \mathrm{IX}, \S 1])$ :

$$
Q_{\alpha}(t)=\frac{1}{2 \pi i} \int_{\Gamma} d \zeta R_{\zeta}\left(T_{\alpha}\right) \exp (-t \zeta)
$$

Here the right-hand side of (9) is a $\|\cdot\|$-convergent Bochner integral with a positively oriented contour $\Gamma$ in the resolvent set of operator $T_{-}$with spectrum of

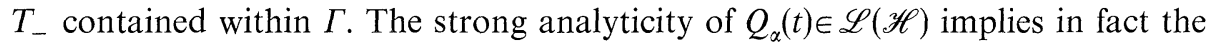
$\|\cdot\|$-analyticity. Therefore, from the estimate

$$
\begin{aligned}
& \left\|1 / t^{\prime}\left(Q_{\alpha}\left(t+t^{\prime}\right)-Q_{\alpha}(t)\right)+T_{\alpha} Q_{\alpha}(t)\right\|_{1} \\
& \quad \leqq\left\|1 / t^{\prime}\left(Q_{\alpha}\left(t^{\prime}\right)-1\right) Q_{\alpha}(t / 2)+T_{\alpha} Q_{\alpha}(t / 2)\right\|\left\|Q_{\alpha}(t / 2)\right\|_{1}
\end{aligned}
$$

for $t, t^{\prime} \in \mathbb{C}_{+}$one gets the existence of

$$
\|\cdot\|_{1}-\lim _{t^{\prime} \rightarrow 0} 1 / t^{\prime}\left(Q_{\alpha}\left(t+t^{\prime}\right)-Q_{\alpha}(t)\right)=-T_{\alpha} Q_{\alpha}(t) \in \mathscr{C}_{1}(\mathscr{H}) .
$$

The limit (10) proves that Gibbs semigroups are $\|\cdot\|_{1}$-holomorphic on $\mathbb{C}_{+}$. Let a connected compact set $G \subset \mathbb{C}_{+}$be such that $G \cap \mathbb{R}_{+}^{1}$ has at least one limit point in $G$. The semigroups $\left\{Q_{\alpha}(t)\right\}_{\alpha \in A}$ are uniformly $\|\cdot\|_{1}$-bounded in $G$ :

$$
\left\|Q_{\alpha}(t)\right\|_{1} \leqq \sup _{t \in G}\left\|Q_{-}(\operatorname{Re} t)\right\|_{1} .
$$

Then $\|\cdot\|_{1}$-compactness of the family $\left\{Q_{\alpha}(t)\right\}_{\alpha \in A}$ on $\mathbb{R}_{+}^{1}$ (Theorem 1 ), inequality (11) and the Vitali convergence theorem (see e.g. [9, III, §2]) imply the $\|\cdot\|_{1}$ compactness of $\left\{Q_{\alpha}(t)\right\}_{\alpha \in A}$ as a family of $\|\cdot\|_{1}$-holomorphic functions in $G$. A little thought shows the same for an arbitrary connected compact set in the right halfplane $\mathbb{C}_{+}$. This means that for the $\|\cdot\|_{1}$-holomorphic on $\mathbb{C}_{+}$family $\left\{Q_{\alpha}(t)\right\}_{\alpha \in A}$ of the Gibbs semigroups there exists a $\|\cdot\|_{1}$-holomorphic on $\mathbb{C}_{+}$function $Q(t)$ and a sequence $\left\{Q_{\alpha_{k}}(t)\right\}_{k=1}^{\infty}$ such that $Q(t)$ is a Gibbs semigroup and $Q_{\alpha_{k}}(t) \stackrel{\|\cdot\|_{1}}{\longrightarrow} Q(t)$ locally uniformly in $\mathbb{C}_{+}$.

Remark 4. As a consequence the set $\left\{Q_{\alpha}(t)\right\}_{\alpha \in A}$ is $\|\cdot\|_{1}$-compact for each $t \in \mathbb{C}_{+}$ (extension of Theorem 1). This may be also deduced from the lemma and Proposition 2 by a little reasoning if one notes that $\left|Q_{a}(t)\right|=Q_{\alpha}(\operatorname{Re} t)$ for $t \in \mathbb{C}$.

Here we discuss no applications of Theorems 1 or 2 in detail, but mention the following two examples.

Example 2. Let $T\left(\Lambda^{N}\right)$ be the self-adjoint kinetic energy operator for an $N$-particle system enclosed in a bounded region $\Lambda \subset \mathbb{R}^{v}$, and $U(N)=\sum_{1 \leqq i<j \leqq N} \Phi\left(x_{i}-x_{j}\right)$ be a semibounded from below symmetric operator of the $N$-particle interaction. If the 
two-body potential $\Phi: \mathbb{R}^{v} \rightarrow \mathbb{R}^{1} \cup\{+\infty\}$ is highly singular near the origin (e.g. hard-core) then the operator $U(N)$ does not need to be a relatively small perturbation for $T\left(\Lambda^{N}\right)$. Therefore, we define a cut-off interaction $U_{L}(N)=\sum_{1 \leqq i<j \leqq N} \Phi_{L}\left(x_{i}-x_{j}\right)$, where $L \geqq 0$ and

$$
\Phi_{L}(x)=\left\{\begin{array}{l}
\Phi(x), x \in\left\{\mathbb{R}^{v}: \Phi(x) \leqq L\right\} \\
L \quad, x \in\left\{\mathbb{R}^{v}: \Phi(x)>L\right\}
\end{array}\right.
$$

Now, the self-adjoint cut-off Hamiltonian $H_{L}\left(\Lambda^{N}\right)$ is well-defined as algebraic sum: $H_{L}\left(\Lambda^{N}\right)=T\left(\Lambda^{N}\right)+U_{L}(N)$ on the domain $D\left(T\left(\Lambda^{N}\right)\right)$, and $H_{L}\left(\Lambda^{N}\right) \leqq H_{+}$where operator $H_{+}=\left(T\left(\Lambda^{N}\right)+U(N)\right)_{F}$ is the Friedrichs extension of the sum $T\left(\Lambda^{N}\right)+U(N)$ from domain $D\left(T\left(\Lambda^{N}\right)\right) \cap D(U(N))$. Thus, the family $\left\{H_{L}\left(\Lambda^{N}\right)\right\}_{L \geq 0}$ satisfies the conditions of the Theorem 1. For $L \rightarrow \infty$ Gibbs semigroups $\left\{\bar{Q}_{L}(t)\right\}_{L \geqq 0}\|\cdot\|_{1}$ converge uniformly in $\mathbb{C}_{+}$to a semigroup $Q(t)$. Its generator $H$ is the sum of operators $T\left(\Lambda^{N}\right)$ and $U(N)$ in the sense of quadratic forms: $H=T\left(\Lambda^{N}\right) \dot{+} U(N)$ (see [10]). Sufficient conditions for $H$ to coincide with $H_{+}$are discussed in $[10,11]$.

Example 3. Let $T$ and $U$ be positive self-adjoint operators in a separable Hilbert space $\mathscr{H}$ and $T$ generates a Gibbs semigroup. If $\mathscr{Q}=\mathscr{Q}(T) \cap \mathscr{Q}(U)$ is dense in $\mathscr{H}$ [here $\mathscr{2}(\cdot)$ corresponds to form domain] then for an arbitrary $\lambda>0$ one can define the self-adjoint operator $H_{\lambda}=T \dot{+} \lambda U$. Hence the family $\left\{H_{\lambda}\right\}_{\lambda>0}$ satisfies the Theorem 1 . When $\lambda \downarrow 0$ the Gibbs semigroups $\left\{Q_{\lambda}(t)\right\}_{\lambda>0}\|\cdot\|_{1}$-converge uniformly in $\mathbb{C}_{+}$to a semigroup $Q(t)$ with generator $H_{0}$ whose associated quadratic form is the closure of the form $t \uparrow \mathscr{Q}$, where $t[\psi]=\left(T^{1 / 2} \psi, T^{1 / 2} \psi\right)$. If the operator $U$ is a sufficiently singular perturbation of $T$ one has $H_{0} \neq T$-Klauder's phenomenon [12]. The operator $H_{0}$ is proved to coincide with $T$ if and only if, the domain 2 is a form-core for operator $T$ (see [5, VIII, §3] and [13]).

Acknowledgements. I am indebted to Dr. N. Angelescu and Prof. A. Uhlmann for generous counsels and discussions. I am grateful to the referee for suggestions concerning some reorganization of the paper. In particular, I owe the referee's comments and proposal to state Propositions 1 and 2 as such. Proposition 1 is a generalization of Theorem 3.5 in Kato's book [5, VIII, §3], and Proposition 2 may be considered as a variation of the Wehrl's Theorem 3 in [14]. I also want to thank the referee for bringing to my attention some relationship between our lemma and Theorem 2.17 in the recent (not yet available to me) Simon's book [15].

\section{References}

1. Schatten, R.: Norm ideals of completely continuous operators. Berlin, Göttingen, Heidelberg: Springer 1960

2. Uhlenbrock, D. : J. Math. Phys. 12, 2503 (1971)

3. Angelescu, N., Nenciu, G., Bundaru, M.: Commun. Math. Phys. 42, 29 (1975)

4. Reed, M., Simon, B.: Methods of modern mathematical physics, Vol. II. Fourier analysis, selfadjointness. New York: Academic Press 1975

5. Kato, T.: Perturbation theory for linear operators. Berlin, Heidelberg, New York: Springer 1966

6. Bogolubov, N.N., jr. : Physica 41, 601 (1969)

7. Maison, H.D.: Commun. Math. Phys. 22, 166 (1971)

8. Zagrebnov, V.A., Brankov, J.G., Tonchev, N.S. : Dokl. Acad. Nauk USSR 225, 71 (1975) 
9. Hille, E., Phillips, R.S.: Functional analysis and semigroups. R.I., Providence: Amer. Math. Soc. Colloquium Publications 1957

10. Zagrebnov, V.A.: Ann. Phys. (N.Y.) 102, 108 (1976)

11. Zagrebnov, V.A., in: Trans. Moscow Math. Soc. Vol. 41, 121 Moscow: Moscow Univ. Press 1980 (in Russian)

12. Klauder, J.R.: Acta Phys. Austr. Suppl. XI, 341 (1973)

13. Simon, B.: J. Funct. Anal. 14, 295 (1973)

14. Wehrl, A.: Reports Math. Phys. 10, 159 (1976)

15. Simon, B.: Trace ideals and their applications. London Math. Soc. Lecture Notes Series 35. Cambridge: Cambridge University Press 1979

Communicated by H. Araki

Received April 28, 1980 